\title{
Interface and contact line motion in a two phase fluid under shear flow
}

\author{
Hsuan-Yi Chen and David Jasnow \\ Department of Physics and Astronomy, University of Pittsburgh, Pittsburgh, Pennsylvania 15260 \\ Jorge Viñals \\ Supercomputer Computations Research Institute, Florida State University, Tallahassee, Florida 32306-4130, and Department \\ of Chemical Engineering, FAMU-FSU College of Engineering, Tallahassee, Florida 32310-6046
}

(May 3, 2018)

\begin{abstract}
We use a coarse grained description to study the steady state interfacial configuration of a two phase fluid under steady shear. Dissipative relaxation of the order parameter leads to interfacial slip at the contact line, even with no-slip boundary conditions on the fluid velocity. This relaxation occurs within a characteristic length scale $l_{0}=\sqrt{\xi D / V_{0}}$, with $\xi$ the (microscopic) interfacial thickness, $D$ an order parameter diffusivity, and $V_{0}$ the boundary velocity. The steady state interfacial configuration is shown to satisfy a scaling form involving the ratio $l_{0} / L$, where $L$ is the width of the fluid layer, for a passive interface, and the capillary number as well for an active interface.
\end{abstract}

We reexamine a classical hydrodynamic problem in which a two-phase fluid is placed in a uniform shear flow by displacing two infinite, parallel planar boundaries at constant relative velocity. Instead of considering the case of two immiscible fluids separated by an infinitely sharp interface, our analysis involves two coexisting fluid phases. The interface, which is diffuse even without shear, is stretched by the flow, but not indefinitely as interfacial slip relieves the increasing shear stress at the three phase junction. A mesoscopic description of the two-phase fluid is introduced to show that diffusive relaxation of the order parameter (e.g., concentration) necessarily contributes to macroscopic interfacial slip. Since in typical situations mutual diffusion occurs on lengths large compared to microscopic (molecular) lengths, it is not necessary to introduce microscopic slip of the fluid velocity near the contact lines. Finally, we also show that in the steady state, the interfacial configuration satisfies a scaling form, provided that the interfacial curvature is small compared to its inverse thickness.

The classical analysis of the contact line profile and motion in partially wetting fluids at low capillary numbers is due to Cox [1], and Dussan and Davis [2]. A matched asymptotic expansion describes both the interfacial configuration and flow field by considering three separate regions. Close to the boundary, the fluid is allowed to slip by invoking a phenomenological relation between the slip velocity and the local shear stress. This relation can also be viewed as introducing a slip length of microscopic dimensions and an effective slip boundary condition for the velocity. Far from the boundaries, capillary forces are balanced by viscous stresses. An intermediate region allows matching between the two. Reviews of this treatment have been given in [3, 4 . Early experiments by Dussan [5] confirmed this picture. More recent experiments by Lichter [6] have addressed the range of capillary numbers within which this solution is a good approximation near the contact line.

If the fluid in question is a binary at coexistence, interfacial deformation induces concentration gradients through a Gibbs-Thomson relation [7]. The resulting diffusive transport leads to effective interfacial slip at the contact line even when the velocity strictly satisfies a noslip boundary condition. Increasing interfacial curvature leads to increasing diffusive transport which eventually balances advection by the flow. A steady state can be maintained. Within a coarse-grained or mesoscopic description of the two phase fluid [8], this balance occurs within a characteristic length scale $l_{0}$, which is the geometric mean of the interfacial thickness and the diffusion length, and hence is a small quantity although not of molecular dimensions. Dissipation within this scale effectively prevents the formation of stress singularities at the contact line. A mesoscopic description of contact line motion has already been given [9], by using the same model equations which we describe below. These authors, however, focused on the limit $l_{0} \rightarrow 0$ (in our notation), which corresponds to effective immiscibility on all scales. The effect that we discuss, however, requires consideration of distances to the boundary small compared to $l_{0}$.

We consider an incompressible binary mixture in two spatial dimensions confined between two planar boundaries of infinite extent located at $x= \pm L / 2$. The bounding planes are displaced with constant velocity $\pm V_{0} / 2 \hat{\mathbf{y}}$. We study the steady state interface configuration in terms of $V_{0}, L$, and the physical parameters of the fluid. Within a coarse grained description of a binary fluid 10 13, the order parameter $\phi$, e.g., concentration, satisfies that $\phi=\phi_{+}>0$ for one phase, and $\phi=\phi_{-}<0$ for the other. The nominal position of the interface is defined as the locus $\phi=0$. The temporal evolution of the order parameter is governed by

$$
\frac{\partial \phi}{\partial t}+\mathbf{v} \cdot \nabla \phi=M \nabla^{2} \mu
$$

where $\mathbf{v}$ is the fluid velocity, the chemical potential $\mu[\phi]=\delta F / \delta \phi$, with $F[\phi]$ the coarse grained free energy functional, and $\delta / \delta \phi$ stands for variational differentia- 
tion. $M$ is a mobility coefficient assumed to be constant. The velocity $\mathbf{v}$ obeys a modified Navier-Stokes equation,

$$
\rho\left(\frac{\partial \mathbf{v}}{\partial t}+\mathbf{v} \cdot \nabla \mathbf{v}\right)=-\nabla p+\mu \nabla \phi+\eta \nabla^{2} \mathbf{v}
$$

where $\rho$ and $\eta$ are the density and shear viscosity of the fluid, assumed to be independent of $\phi$, and $p$ is the pressure. The second term on the right hand side of Eq. (2) incorporates the effects of capillarity. We choose the standard free energy $F[\phi]=\int d V\left(\frac{K}{2}|\nabla \phi|^{2}+f(\phi)\right)$, with $K$ constant. The first term represents the excess free energy due to spatial inhomogeneities of $\phi$, and $f(\phi)$ is the local part of the free energy density. Generalizations involving, say, higher gradients or forcing due to short-ranged wall interactions can be incorporated.

Several macroscopic physical quantities follow from this coarse grained description [14,7]. The surface tension is given by $\sigma=\int_{-\infty}^{\infty} K\left[\partial \phi_{0} / \partial y\right]^{2} d y$, where $\phi_{0}$ is the equilibrium order parameter profile at coexistence (we take $\mu\left(\phi_{0}\right)=0$ in what follows). Furthermore, the chemical potential at a gently curved interface with radius of curvature $R_{c}$, relative to its value at coexistence, is $\mu=-\sigma / R_{c} \Delta \phi$, where $\Delta \phi=\phi_{+}-\phi_{-}$is the miscibility gap. Another important quantity is the (finite) interfacial thickness which is proportional to $\xi=\sqrt{K \chi}$ where $\chi^{-1}=\left(\partial^{2} f / \partial \phi^{2}\right)_{\phi_{ \pm}}$. Since we assume that the fluid is not too close to criticality, $\xi$ is of molecular dimensions. Finally, we focus exclusively on moderate shear rates, such that $\xi / R_{c} \ll 1$, a limit commonly realized in practice.

We begin by illustrating the effect of the shear on the interface through dimensional analysis. If the interfacial mean curvature is $1 / l$, then the local deviation of $\mu$ from coexistence is proportional to $1 / l$. We therefore choose the following dimensionless (barred) quantities: $\mu=\frac{\sigma}{l \Delta \phi} \bar{\mu}, \phi=(\Delta \phi) \bar{\phi}$, and $\mathbf{v}=V_{0} \overline{\mathbf{v}}$. In steady state, $P e \overline{\mathbf{v}} \cdot \bar{\nabla} \bar{\phi}=\bar{\nabla}^{2} \bar{\mu}$, where $P e=V_{0} \Delta \phi^{2} l^{2} / M \sigma$. On small scales $(l \sim \xi), P e$ becomes very small and hence $\bar{\nabla}^{2} \bar{\mu}=0$ defines the equilibrium order parameter profile in the absence of shear. Diffusion and miscibility are important. If, on the other hand, $l \sim L \rightarrow \infty$, then $\bar{\nabla} \bar{\phi} \cdot \overline{\mathbf{v}}=0$, corresponding either to a homogeneous configuration, or to an interface parallel to the flow. Miscibility effects are not important at this scale. There exists, however, an intermediate length scale $l_{0}=\sqrt{M \sigma / V_{0} \Delta \phi^{2}}$ for which $P e=1$. Advection now balances order parameter diffusion along the interface driven by chemical potential gradients; this is the length scale of interest. Given the definition of the interfacial thickness $\xi$, we can write $l_{0} \propto \sqrt{\xi D / V_{0}}$, where $D=M / \chi$ is a diffusion coefficient. Hence this new length scale is the geometric mean of the interfacial thickness and a diffusion length $D / V_{0}$. For a typical system, $D \sim 10^{-5} \mathrm{~cm}^{2} / \mathrm{s}, \xi \sim 10 \AA$, and for a wall velocity $V_{0} \sim 1 \mathrm{~cm} / \mathrm{s}, l_{0} \approx 10 \mathrm{~nm}$.

We next present an approximate analytic treatment that is valid for length scales larger than $\xi$. We find a scaling solution for the steady state interfacial profile for both passive interfaces (neglecting capillarity induced backflow, i.e., model B in the critical dynamics lexicon) and active interfaces. We then present a numerical solution of the full set of governing equations to validate the scaling forms derived, at least within the range of parameters which is numerically accessible.

For a passive interface, the velocity field is given by $\mathbf{v}_{\mathbf{s}}(\mathbf{r})=x V_{0} / L \hat{\mathbf{y}}$. Equation (11) in steady state is written in integral form,

$$
M \mu(\mathbf{r})=\int d r^{\prime} G\left(\mathbf{r}, \mathbf{r}^{\prime}\right)[\mathbf{v} \cdot \nabla \phi]_{r^{\prime}},
$$

where $G\left(\mathbf{r}, \mathbf{r}^{\prime}\right)$ is the Green's function of the Laplacian operator satisfying Neumann boundary conditions at $x=$ $\pm L / 2$ 15. In two dimensions the Green's function is dimensionless. Let the position of the nominal interface be $\mathbf{r}_{I}=y_{I}(x) \hat{\mathbf{y}}+x \hat{\mathbf{x}}$, where $\hat{\mathbf{x}}$ and $\hat{\mathbf{y}}$ are unit vectors. When radii of curvature are large compared to $\xi$, one can make the standard approximation, $\nabla \phi \simeq(\Delta \phi) \delta(\mathbf{r}-$ $\left.\mathbf{r}_{I}\right) \hat{\mathbf{n}}$, and $\phi=\phi_{ \pm}$. [16] Using now standard methods (see, e.g., Ref. [7,16]), one multiplies Eq. (3) by $\partial \phi / \partial g$ $(g=\mathbf{r} \cdot \vec{\nabla} \phi /|\nabla \phi|)$ and integrates $g$ across the interface to derive an equation that depends on the coordinates of the interface alone. The result is

$$
\frac{1}{R(X)}=-\left(\frac{l_{0}}{L}\right)^{-2} \int_{-1 / 2}^{1 / 2} d X^{\prime} X^{\prime} G\left(\mathbf{R}_{\mathbf{I}}, \mathbf{R}_{\mathbf{I}}^{\prime}\right)
$$

where we have now scaled lengths by $L\left(R=R_{c} / L\right.$, $X=x / L, Y=y / L)$, and $\mathbf{R}_{\mathbf{I}}(X)=\mathbf{r}_{I}(x) / L$ is the scaled location of the interface. This equation is an integrodifferential equation for the interface coordinate $Y_{I}(X)$. Boundary conditions need to be imposed at a distance of order $\xi$ from the boundary. At this scale the shear is negligible so that the equilibrium order parameter profile is adequate. For unforced conditions, this leads to $Y_{I}^{\prime}=0$, although other situations can be accommodated. 17 According to Eq. (4), the curvature of the interface satisfies the following scaling relation

$$
R^{-1}(X)=f_{1}\left(\left(l_{0} / L\right)^{2}, X\right) .
$$

Hence, the interface configuration is solely determined by the dimensionless number $\left(l_{0} / L\right)^{2}$, and by the equilibrium boundary conditions on the order parameter at $x= \pm L / 2$.

Since Eq. (4) is expected to be valid only on length scales large compared to $\xi$, we have numerically solved Eq. (11) to verify the above scaling form, as well as to determine the approach to this scaling form as $l_{0} / \xi>>1$. The details of the numerical algorithm have been described elsewhere 10]. Briefly, the fluid is in a rectangular domain $-L / 2 \leq x \leq L / 2$ and $-3 L / 2 \leq y \leq 3 L / 2$, with boundary conditions $\hat{\mathbf{n}} \cdot \nabla \phi=0$ and $\hat{\mathbf{n}} \cdot \nabla\left(\nabla^{2} \phi\right)=0$ at $x= \pm L / 2$, and $\phi=\phi_{ \pm}$at $y= \pm 3 L / 2$. (The corresponding equilibrium contact angle is $\pi / 2$; the boundary conditions also ensure no flux of $\phi$ through the lateral 
walls.) The interface is initially located at $y=0$, and we integrate Eq. (11) forward in time until a stationary configuration is reached. The local part of free energy density is the standard double well potential [10], and a square grid has been used of side no larger than $\xi / 3$ so that the relative error in the location of the interface is $5 \%$ or less. Fig. 1 shows the interfacial configuration for a range of $l_{0} / \xi$. The curves tend to superpose as $l_{0} / \xi$ becomes larger for fixed $l_{0} / L$, supporting the scaling prediction.

For the range of parameters considered, the interfacial displacement away from planarity is small. Therefore we also obtain a perturbative solution of Eq. (4). Direct substitution of the Green's function [15] into Eq. (4) leads to,

$Y_{I}(X)=\frac{2}{\pi^{5}}\left(\frac{L}{l_{0}}\right)^{2} \sum_{n=1}^{\infty} \frac{(-1)^{n-1}}{(2 n-1)^{5}} \sin ((2 n-1) \pi x)$

at lowest order in $Y_{I}$. Note that the term corresponding to $n=1$ dominates so that the correct dimensionless parameter for the expansion should be $\left(L / l_{0}\right)^{2} \pi^{-5}$. We next define $\delta=-1 / 2+X$ and find the curvature of the interface near the boundary, $R^{-1}(-1 / 2+\delta)=-R_{w}^{-1}+$ $\frac{1}{4 \pi}\left(L / l_{0}\right)^{2} \times\left[(3-2 \ln (\pi / 2)) \delta^{2}-2 \delta^{2} \ln \delta\right]+\ldots$, where terms $O\left(\left(L / l_{0}\right)^{4}\right)$ and $O\left(\delta^{4}\right)$ have been neglected. The constant $R_{w} \approx(0.426 / 2 \pi)\left(L / l_{0}\right)^{2}$. Note that there is a weak singularity as $\delta \rightarrow 0$ (a divergent second derivative), that can be traced back to the factor $(2 n-1)^{-5}$ in Eq.(6). The perturbative solution for $Y_{I}$ is also shown in Fig. 1. The singularity in the second derivative of the curvature is much too weak to be observed directly.

We next consider an active interface and allow for the additional flow induced by order parameter variations. Boundary conditions for Eq. (2) are no-slip at the solid boundaries, $\mathbf{v}= \pm V_{0} / 2 \hat{\mathbf{y}}$ at $x= \pm L / 2$, and a far field velocity approaching $\mathbf{v}_{\mathbf{s}}$. The steady state configuration of $\mu$ is again given by Eq. (3), but $\mathbf{v}$ needs to be determined self-consistently. Under the same assumptions used to derive the interface equation in the case of a passive interface and neglecting the inertial term, one finds [7, 16],

$$
\begin{aligned}
& \frac{1}{R(X)}=\left(\frac{l_{0}}{L}\right)^{-2} \int_{-1 / 2}^{1 / 2} d X^{\prime} G\left(\mathbf{R}_{I}, \mathbf{R}_{I}^{\prime}\right)\left[-X^{\prime}+\right. \\
& \left.\frac{1}{C a} \frac{d S}{d X^{\prime}} \int_{-1 / 2}^{1 / 2} d X^{\prime \prime} \frac{d S}{d X^{\prime \prime}} n_{i}^{\prime} T_{i j}\left(\mathbf{R}_{I}^{\prime}, \mathbf{R}_{I}^{\prime \prime}\right) n_{j}^{\prime \prime} \frac{1}{R\left(X^{\prime \prime}\right)}\right]
\end{aligned}
$$

Repeated indices imply summation, and $T_{i j}$ is the Oseen tensor 18. A capillary number has been defined as $C a=\eta V_{0} / \sigma, n_{i}^{\prime}=n_{i}\left(X^{\prime}\right)$ is the $i$ th component of the unit normal to the interface at $X^{\prime}$, and $d S(X) / d X=$ $\sqrt{1+\left(d Y_{I}(X) / d X\right)^{2}}$. This interface equation, in turn, suggests the following scaling form,

$$
R^{-1}(X)=f_{2}\left(C a,\left(l_{0} / L\right)^{2}, X\right)
$$

In the limit $\mathrm{Ca} \rightarrow \infty$, the passive case is recovered (Eq. (4)).

We have also verified this scaling form by direct numerical solution of Eqs. (11) and (2) subject to the boundary conditions given: no-slip at $x= \pm L / 2$, and $\mathbf{v}=\mathbf{v}_{\mathbf{s}}$ at $y= \pm 3 L / 2$. Figure 2 shows the results obtained for fixed $l_{0} / L$ and $C a$ and a range of values of $l_{0} / \xi$. Again, these results are consistent with the scaling forms derived. Note that the values of $l_{0} / L$, and $l_{0} / \xi$ are the same in both Figs. 1 and 2, but the interface deflection in Fig. 2 is much smaller than that in Fig. 1. This is a consequence of the additional dissipation mechanism in the fluid and the induced flow. The small values of $Y_{I}$ in the numerical solution suggest that $Y_{I}$ is proportional to $V_{0}$, as can be seen from the inset in Fig. 2.

We now compare our results with previous studies of immiscible fluids based on a macroscopic description [1]3]. Equation (8) can be written in the following scaling form $l_{0} / R_{c}\left(x / l_{0}\right)=\tilde{f}_{2}\left(C a, l_{0} / L, x / l_{0}\right)$. Now choose the origin such that the walls are located at $x=0$, and $L$. In the limit $x / L \ll 1$ but $x / l_{0} \gg 1$, one expects that the dependence of the curvature on both $L$ and $l_{0}$ is weak. If the leading behavior is such that the curvature is independent of both $L$ and $l_{0}$, then the scaling function reduces to $\tilde{f}_{2} \sim \tilde{f}_{3}(C a) l_{0} / x$. Using the fact that $\tilde{f}_{3}(C a) \simeq A_{0} C a$ [19], this relation can be integrated to yield $\frac{d y_{I}}{d x}=A_{0} C a \ln \frac{x}{x_{0}}+B_{0}$ where $A_{0}$ and $B_{0}$ are constant, and $x_{0} / l_{0} \gg 1$. This "outer solution", valid for small $C a$ and $x / l_{0} \gg 1$, has the same functional form as the immiscible case [1]. There, the expansion parameter in the matched asymptotic expansion is $\epsilon=s / L$, where $s$ is constant and of molecular dimensions. In the present case, however, the inner region is determined by $l_{0}$, which is a function of the boundary velocity. Therefore a similar matched asymptotic expansion to derive the macroscopic interfacial profile for a two phase fluid would lead to a qualitatively different behavior. In particular, note that as $V_{0}$ becomes small, nonlocal diffusive coupling between distant regions of the interface becomes important. We note finally that our conclusions are based on the scaling form derived from Eq. (7), which is valid only for $x \gg \xi$, although it has also been numerically validated for the range of parameters that we can explore computationally.

To summarize, we have studied the steady state interfacial configuration in a partially miscible two-phase fluid under steady shear. Including mutual diffusion and miscibility naturally introduces a cutoff, physical in nature, which eliminates stress singularities. An approximate integro-differential equation for the steady state interface configuration has been derived, valid at distances larger than the interfacial thickness $\xi$. For a passive interface, the configuration satisfies a scaling form that depends only on the ratio $l_{0} / L$, where $l_{0}=\sqrt{\xi D / V_{0}}$. We then argue that the length scale $l_{0}$, which is much larger than molecular dimensions but not necessarily macroscopic, defines the scale over which effective interface slip occurs purely due to diffusive relaxation of the order parameter. 
Similar results are obtained for an active interface, with the scaling function now depending on both $l_{0} / L$ and the capillary number $C a=\eta V_{0} / \sigma$. Numerical solutions of the full dynamical equations, Eqs. (1)- (2), support both scaling forms. Our results indicate that the slip mechanism of an interface separating coexisting fluids is qualitatively different than in the immiscible case. Experiments involving very small $\mathrm{Ca}$ on coexisting fluids would be useful to verify our results.

DJ is grateful for the support of the NSF under DMR9217935. JV is supported by the U.S. Department of Energy, contract No. DE-FG05-95ER14566, and also in part by the Supercomputer Computations Research Institute, which is partially funded by the U.S. Department of Energy, contract No. DE-FC05-85ER25000.

[1] R. G. Cox, J. Fluid Mech., 168, 169, (1986).

[2] E. B. Dussan V, and S. H. Davis, J. Fluid Mech. 173, 115 (1986), and reference therein.

[3] P. G. de Gennes, Rev. Mod. Phys. 57, 827, (1985)

[4] A. Oron, S. H. Davis, and S. G. Bankoff, Rev. Mod. Phys. 69, 931 (1997).

[5] E.B. Dussan V., J. Fluid Mech. 209, 191 (1989)

[6] S. Lichter, Proc. $4^{\text {th }}$ NASA Microgravity Fluid Physics and Transport Phenomena Conference, p. 135 (1998) (http://www.ncmr.org/events/fluids1998.html).

[7] A.J. Bray, Adv. Phys., 43, 357, (1994).

[8] P. C. Hohenberg, and B. I. Halperin, Rev. Mod. Phys. 49, 435, (1977); and A. Onuki and K. Kawasaki, Ann. Phys. (N.Y.) 121456 (1979).

[9] P. Seppecher, Int. J. Engng. Sci. 34, 977 (1996); and D. Jacqmin, unpublished.

[10] D. Jasnow and J. Viñals, Phys. Fluids 8 (3), 660 (1996).

[11] R. Bhagavatula, D. Jasnow, and T. Ohta, J. Stat. Phys. 88, 1013, (1997).

[12] M. Gurtin, D. Polignone, and J. Viñals, Math. Mod. and Methods in Appl. Sci.,6, 815 (1996).

[13] D.M. Anderson, G.B. McFadden and A.A. Wheeler, Annu. Rev. Fluid Mech. 30, 139 (1998).

[14] D. Jasnow, Rep. Prog. Phys., 47, 1095, (1984).

[15] The explicit form of the Green's function is,

$$
\begin{aligned}
G\left(\mathbf{r}, \mathbf{r}^{\prime}\right)= & \left|y-y^{\prime}\right| / 2 L \\
& +\ln \left[\left(1+2 P \cos \left(\pi\left(x+x^{\prime}\right) / L\right)+P^{2}\right)\right] / 4 \pi \\
& +\ln \left[\left(1-2 P \cos \left(\pi\left(x-x^{\prime}\right) / L\right)+P^{2}\right)\right] / 4 \pi,
\end{aligned}
$$

where $P=\exp \left(-\pi\left|y-y^{\prime}\right| / L\right)$.

[16] K. Kawasaki and T. Ohta, Physica A 118, 175, (1983).

[17] Introducing wall potentials into the coarse grained free energy will allow different values of the equilibrium contact angle.

[18] See, e.g., C. Pozrikidis, Boundary integral and singularity methods for linearized viscous flow, (Cambridge University Press, New York, 1992).
[19] When $l_{0} / L$ is negligible, Eq. (7) can be written,

$\int_{-1 / 2}^{1 / 2} d X^{\prime} G\left[-C a X^{\prime}+\int_{-1 / 2}^{1 / 2} d X^{\prime \prime} \frac{d S}{d X^{\prime}} \frac{d S}{d X^{\prime \prime}} n_{i}^{\prime} T_{i j} n_{j}^{\prime \prime} \frac{1}{R^{\prime \prime}}\right]=0$.

To lowest order in $C a$, this leads to $0=-C a X+$ $\int_{-1 / 2}^{1 / 2} d X^{\prime} T_{y y}\left(X \hat{\mathbf{i}}, X^{\prime} \hat{\mathbf{i}}\right) / R\left(X^{\prime}\right)$; hence the curvature is proportional to $C a$ when $C a$ is small.

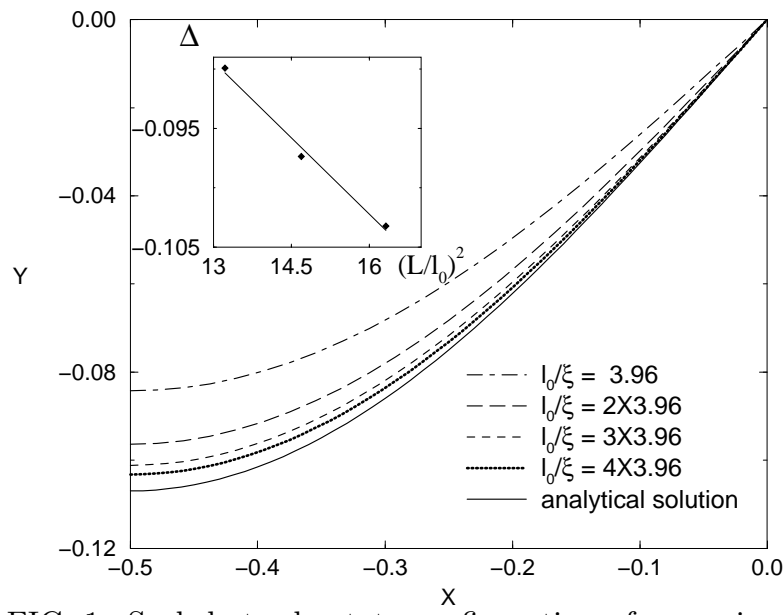

FIG. 1. Scaled steady state configuration of a passive interface for the values of $l_{0} / \xi$ listed, and $L=4.04 l_{0}$. The inset shows the maximum interface deflection $\Delta$ as a function of $\left(L / l_{0}\right)^{2}$ at $l_{0} / \xi=4 \times 3.96$.

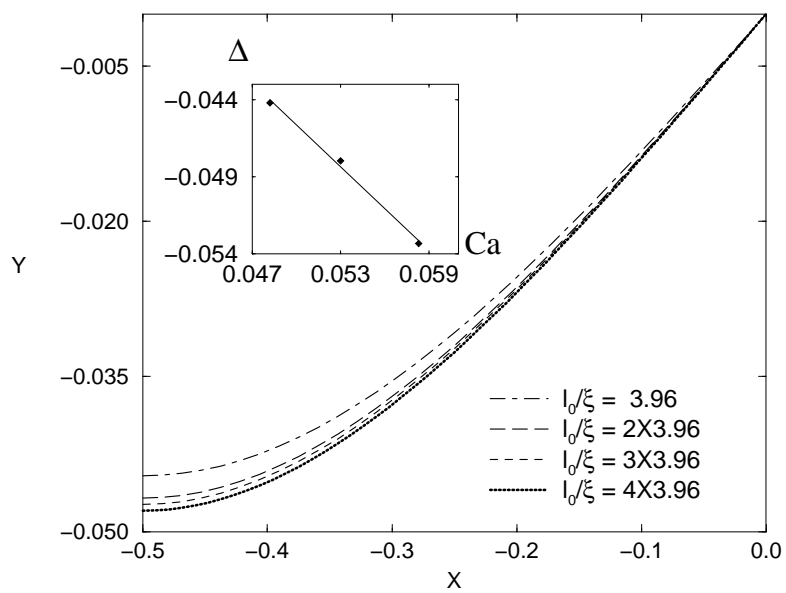

FIG. 2. Scaled steady state configuration of an active interface for the values of $l_{0} / \xi$ listed, $L=4.04 l_{0}$ with $C a=0.053$. The inset reveals the maximum interface deflection $\Delta$ for different wall velocities (i.e., different $C a$ ) with fixed $L / \xi$ and $l_{0}^{2} \mathrm{Ca}$. 